\title{
Characterization of bone marrow-derived mesenchymal stem cells from dimethyloxallyl glycine-preconditioned mice: Evaluation of the feasibility of dimethyloxallyl glycine as a mobilization agent
}

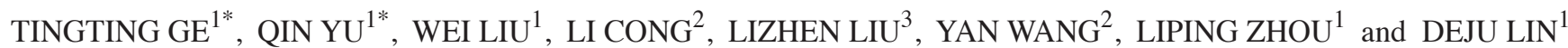 \\ ${ }^{1}$ Department of Bioengineering, College of Life Science; ${ }^{2}$ Department of Pediatrics, The First Clinical Medical College, \\ Zhejiang Chinese Medical University, Hangzhou, Zhejiang $310053 ;{ }^{3}$ Bone Marrow Transplantation Center, \\ The First Affiliated Hospital, Medical School of Zhejiang University, \\ Hangzhou, Zhejiang 310003, P.R. China
}

Received March 30, 2015; Accepted January 27, 2016

DOI: $10.3892 / \mathrm{mmr} .2016 .4945$

\begin{abstract}
The prolyl hydroxylase inhibitor dimethyloxallyl glycine (DMOG) has been increasingly studied with regards to stem cell therapy. Previous studies have demonstrated that endogenous mesenchymal stem cells (MSCs) may be mobilized into peripheral circulation by pharmaceutical preconditioning. In addition, our previous study confirmed that DMOG, as a novel mobilization agent, could induce mouse/rat MSC migration into peripheral blood circulation. Therefore, the present study conducted studies to characterize bone marrow-derived MSCs (BM-MSCs) collected from mice following DMOG intraperitoneal injection. The surface antigen immune
\end{abstract}

Correspondence to: Professor Qin Yu, Department of Bioengineering, College of Life Science, Zhejiang Chinese Medical University, 548 Binwen Road, Hangzhou, Zhejiang 310053, P.R. China

E-mail: qinyu3587@126.com

*Contributed equally

Abbreviations: DMOG, dimethyloxallyl glycine; MSCs, mesenchymal stem cells; BM-MSCs, bone marrow-derived mesenchymal stem cells; DBM-MSCs, bone marrow-derived mesenchymal stem cells from dimethyloxallyl glycine-preconditioned mice; NBM-MSCs, bone marrow-derived mesenchymal stem cells from normal saline-treated mice; CCK-8, Cell Counting kit-8; ELISA, enzyme-linked immunosorbent assay; TGF, transforming growth factor; PDGF, platelet-derived growth factor; HIF-1 $\alpha$, hypoxia-inducible factor-1 $\alpha$; ICR, Male Institute of Cancer Research; NS, normal saline; DMEM/F-12, Dulbecco's modified Eagle's medium/Ham's F-12; FBS, fetal bovine serum; PBS, phosphate-buffered saline; DMEM-LG, Dulbecco's modified Eagle's medium-low glucose; SDF-1 $\alpha$, stromal cell-derived factor-1 $\alpha$; PPAR $\gamma$, peroxisome proliferator-activated receptor- $\gamma$

Key words: bone marrow-derived mesenchymal stem cells, dimethyloxallyl glycine, immune phenotype, proliferation, multilineage differentiation, migration, paracrine, mobilization agent phenotype, differentiation capability, proliferative ability, migratory capacity and paracrine capacity of the BM-MSCs collected from DMOG-preconditioned mice (DBM-MSCs) or normal saline-treated mice (NBM-MSCs) were evaluated by means of flow cytometry, differentiation induction, Cell Counting kit-8, Transwell assay and enzyme-linked immunosorbent assay, respectively. Compared with NBM-MSCs, DBM-MSCs displayed a similar immune phenotype and multilineage differentiation capability, reduced proliferative ability and migratory capacity, and similar transforming growth factor and platelet-derived growth factor secretion capacity. These results provide a novel insight into the biological properties of BM-MSCs from mice preconditioned with DMOG. DBM-MSCs exhibited slightly distinct characteristics to NBM-MSCs; however, they may have therapeutic potential for future stem cell therapy. In addition, the present study suggested that DMOG may be used as a novel mobilization agent in future clinical trials as no adverse effects were observed.

\section{Introduction}

The prolyl hydroxylase inhibitor dimethyloxallyl glycine (DMOG) is a type of hypoxia-mimetic agent, which has been increasingly studied with regards to stem cell therapy. DMOG, which is a small molecular drug, is a cell-permeable prolyl-4-hydroxylase inhibitor. At normal oxygen tension, DMOG is able to inhibit the effects of hypoxia-inducible factor prolyl hydroxylase, and stabilize the expression of hypoxia-inducible factor- $1 \alpha(\mathrm{HIF}-1 \alpha)$ in cells (1), thus mediating the function of signaling pathways associated with cellular or tissue alterations and repair.

A previous study regarding DMOG application reported that DMOG was able to induce cardioprotective effects via HIF-1 $\alpha$ stabilization in rabbits (2). In the past decade, studies on DMOG have gradually increased with regards to its role in various cells and tissues. In recent studies, DMOG has been successfully used to increase bone healing capacity (3), attenuate renal injury in the remnant kidney model (4), induce angiogenesis in ischemic skeletal muscle (5), promote vascularization in the arterovenous loop via HIF-1 $\alpha$ upregulation (6), 
and provide neuroprotection in a middle cerebral artery occlusion model (7). Furthermore, it has been reported that DMOG may have an important role in mesenchymal stem cell (MSC) transplantation therapy for the functional recovery of ischemic heart disease (8).

MSCs are plastic-adherent, fibroblast-like adult stem cells, which possess the capacity to self-renew and differentiate into numerous types of cells, including adipocytes, osteoblasts, chondroblasts, myoblasts and neuron-like cells (9-11). In addition, MSCs can be obtained from various connective tissue sources, including bone marrow (12), adipose (13), dermal tissue (14), synovial fluid (15), deciduous teeth $(16,17)$ and umbilical cord blood (18). Following adequate stimulation, stem and progenitor cells may leave the cell niche and enter the peripheral blood, which is termed stem cell mobilization (19). Although it has been confirmed that allograft MSCs, following in vitro amplification, can repair numerous types of tissue damage, it has been suggested that in vitro-amplified MSCs may induce the development of tumors (20), thus arousing doubts regarding the safety of allograft MSCs. Therefore, increasing attention has been paid with regards to the repairing effect of autologous MSCs. Unfortunately, the strategy by which endogenous MSCs are used to treat tissue damage is limited by the paucity of circulating MSCs in peripheral blood. Recently, pharmacological preconditioning has been proposed hypothetically and experimentally as an efficient approach for modification and improvement in the function of various organs, tissues and cells. Granulocyte colony-stimulating factor (G-CSF) is a potent stimulator of hematopoietic stem cells mobilization. Numerous studies have attempted to use G-CSF to induce MSC mobilization into the circulation, however, disappointing results have been obtained (21-23). Recent studies have reported that recombinant wingless-related integration site (Wnt)3a (24), insulin-like growth factor-1 + AMD3100 (25) and $\mathrm{LiCl}$ (26) mobilize MSCs into the circulation and promote the proliferation and differentiation ability of PB-MSCs. Our previous study confirmed that DMOG was able to mobilize MSCs into peripheral blood circulation (27). Peripheral blood-MSCs were demonstrated to have weaker proliferation and migration ability and similar multilineage differentiation potential when compared with bone marrow-MSCs and the mechanism underlying MSCs mobilization was investigated (28).

MSC mobilization is commonly measured using the fibroblast-colony forming unit assay and flow cytometry. Our previous study demonstrated that compared with normal saline-treated mice, the number of colony forming units and percentage of cluster of differentiation (CD) $90^{+} / \mathrm{CD}^{4} 5^{-}$cells in the peripheral blood was significantly increased in mice treated with DMOG (27).

Whether DMOG may be feasible as a novel mobilization agent remains to be elucidated. Since bone marrow is one of the most important sources of mobilized peripheral blood MSCs, the present study conducted preliminary studies to characterize BM-MSCs collected from mice following DMOG intraperitoneal injection. The biological properties of BM-MSCs from DMOG preconditioned-mice (DBM-MSCs), including cell morphology, immune phenotype, multilineage differentiation, proliferation, migration and paracrine capacity, were investigated and compared with the properties of BM-MSCs from normal saline-treated mice (NBM-MSCs). The results may provide an experimental basis for the application of DMOG as a novel mobilization agent in future clinical trials.

\section{Materials and methods}

Animals and DMOG preconditioning. The present study was approved by the ethics committee of Zhejiang Chinese Medical University (Hangzhou, China). Male Institute of Cancer Research (ICR) mice (age, 8-10 weeks) were purchased from the Zhejiang Chinese Medical University Animal Center (Hangzhou, China; Laboratory Animal Certificate: SCXK 2008-0115). All procedures related to the care of animals were performed according to the National Institutes of Health Guide for the Care and Use of Laboratory Animals (29).

ICR mice were randomly assigned into the normal saline control group (NS group) or the DMOG preconditioning group (DMOG group; $n=5 /$ group). Mice of the DMOG group received an intraperitoneal injection of DMOG $(40 \mathrm{mg} / \mathrm{kg}$ in $0.5 \mathrm{ml}$ normal saline; Cayman Chemical Company, Ann Arbor, MI, USA) for 7 consecutive days, whereas mice in the NS group received $0.5 \mathrm{ml}$ normal saline. The DMOG dose was chosen according to our previous study (27).

Isolation and culture of BM-MSCs. Following DMOG or normal saline preconditioning, mice were sacrificed by cervical dislocation and BM-MSCs were obtained from the mice femur and tibia, as previously described (30). Briefly, muscles and adherent tissue were detached, and the epiphyses were removed. The whole bone marrow plugs were flushed using a 25-gauge needle and a $1.0 \mathrm{ml}$ syringe loaded with Dulbecco's modified Eagle's medium/Ham's F-12 (DMEM/F-12; Gibco; Thermo Fisher Scientific, Inc., Waltham, MA, USA) supplemented with $10 \%$ fetal bovine serum (FBS; Gibco; Thermo Fisher Scientific, Inc.) and 1\% penicillin-streptomycin (HyClone; Thermo Fisher Scientific, Inc., Logan, UT, USA). Harvested marrow cells were centrifuged, resuspended, counted, and cultured as described previously (30). All non-adherent cells were removed by media replacement after $24 \mathrm{~h}$. Subsequently, the medium was replaced every 3-4 days. Upon reaching 80-90\% confluence, the primary cells were trypsinized (0.25\% trypsin-EDTA; Gibco; Thermo Fisher Scientific, Inc.), resuspended in complete culture medium, and subcultured at a 1:2 ratio. BM-MSCs from passages 3-4 were used in the subsequent experiments.

For identification of BM-MSCs, a colony-forming unit fibroblast assay was conducted, and cell surface markers CD90 and CD45 were detected by flow cytometry. The detailed characteristics were confirmed in our previous study (27). CD $44^{+} / \mathrm{CD} 90^{+} / \mathrm{CD} 45^{-}$cells were detected and selected in the present study.

Flow cytometry assay. To detect the effects of DMOG on the immune phenotype of BM-MSCs, flow cytometry was conducted. The fourth generation NBM-MSCs and DBM-MSCs were suspended in phosphate-buffered saline (PBS) at a concentration of $1 \times 10^{6}$ cells $/ \mathrm{ml}$ in five tubes. Fluorescein isothiocyanate (FITC) and phycoerythrin (PE) isotype controls were added into the two tubes as controls, whereas $2 \mu \mathrm{l} \mathrm{CD} 44-F I T C, 5 \mu \mathrm{l}$ CD90-PE and $2 \mu \mathrm{l} \mathrm{CD} 45-\mathrm{FITC}$ 
were added into the three remaining tubes, respectively (all obtained from Abcam (Hong Kong) Ltd., Hong Kong, China). Following a $30 \mathrm{~min}$ incubation at room temperature the cells were washed twice with PBS. Subsequently, a FACSCalibur ${ }^{\mathrm{TM}}$ flow cytometer (BD Biosciences, Franklin Lakes, NJ, USA) and Cell Quest software (version 7.5.3; BD Biosciences) were used to test and analyze the results.

Multilineage differentiation assay. The multilineage differentiation potential of the NBM-MSCs and DBM-MSCs was evaluated in detail, as described in the following subsections.

Adipogenic differentiation. NBM-MSCs and DBM-MSCs were seeded in 6-well plates at a density of $2 \times 10^{4} \mathrm{cells} / \mathrm{cm}^{2}$. Once the cells reached $90-100 \%$ confluence, ICR mouse MSC adipogenic differentiation medium [Cyagen Biosciences (Guangzhou), Inc., Guangzhou, China] was added, and the induction process was conducted according to the manufacturer's protocol. Cells were then stained with Oil Red O solution [Cyagen Biosciences (Guangzhou), Inc.) and were observed under an inverted fluorescence microscope (ECLIPSE TE2000-S; Nikon Corporation, Tokyo, Japan).

Osteogenic differentiation. NBM-MSCs and DBM-MSCs $\left(1 \times 10^{4}\right.$ cells $\left./ \mathrm{cm}^{2}\right)$ were seeded in 6-well plates in DMEM-low glucose (DMEM-LG; Gibco; Thermo Fisher Scientific, Inc.) supplemented with $10 \%$ FBS, $0.1 \mu \mathrm{M}$ dexamethasone, $10 \mathrm{mM} \beta$-glycerol phosphate and $50 \mu \mathrm{M}$ ascorbic acid (all Sigma-Aldrich, St. Louis, MO, USA), and the medium was replaced every 3 days. Cell morphology was observed under an inverted fluorescence microscope (ECLIPSE TE2000-S). Von Kossa (Sigma-Aldrich) staining was used to reveal mineralized areas.

Chondrogenic differentiation. NBM-MSCs and DBM-MSCs were resuspended in serum-free DMEM-high glucose (Gibco; Thermo Fisher Scientific, Inc.) containing insulin-transferrin-selenium-A (Gibco; Thermo Fisher Scientific, Inc.), $0.1 \mu \mathrm{M}$ dexamethasone and $200 \mu \mathrm{M}$ ascorbic acid, and were plated in 6 -well plates at a density of $5 \times 10^{4}$ cells $/ \mathrm{ml}$. Subsequently, $10 \mathrm{ng} / \mathrm{ml}$ transforming growth factor (TGF)- $\beta 1$ and TGF- $\beta 3$ (PeproTech, Rocky Hill, NJ, USA) were added to the cells. The medium was changed every 3 days, and cell factors were renewed each time. Cells were stained with Toluidine Blue (Sigma-Aldrich) after 21 days and visualized under an ECLIPSE TE2000-S microscope.

Neuronal differentiation. The following neuronal differentiation induction media were used in the present study: Pre-induction medium (DMEM-LG containing $1 \mathrm{mM}$ $\beta$-mercaptoethanol and $20 \%$ FBS), and induction medium (serum-free DMEM-LG containing $5 \mathrm{mM} \beta$-mercaptoethanol). NBM-MSCs and DBM-MSCs were plated at a density of $1 \times 10^{5}$ cells/well on $18 \times 18 \mathrm{~mm}$ slides on 6 -well plates. Once the cells reached $\sim 70 \%$ confluence, differentiation was successively induced by incubation with pre-induction medium for $24 \mathrm{~h}$, followed by incubation with induction medium for 3-6 $\mathrm{h}$.

Immunofluorescence. Immunofluorescence assay was used to identify the expression of the nerve cell-specific marker
Nestin, and the astrocyte-specific marker glial fibrillary acidic protein (GFAP). Briefly, slides were gently washed twice with PBS, and were fixed with immune dyeing fixative (Hangzhou Dawen Biotec Co., Ltd., Hangzhou, China) for $10 \mathrm{~min}$. Subsequently, immune dyeing wash buffer (Hangzhou Dawen Biotec Co., Ltd.) was used to wash the slides, and the slides were blocked with immune dyeing block buffers (Hangzhou Dawen Biotec Co., Ltd.) for 60 min. The remaining liquid was aspirated, and the slides were then incubated with polyclonal rabbit anti-mouse Nestin (1:50; Santa Cruz Biotechnology, Inc., Dallas, TX, USA; cat. no. sc-20978) and GFAP (1:100; Santa Cruz Biotechnology, Inc.; cat. no. sc-9065) primary antibodies for $90 \mathrm{~min}$ at room temperature. Following three washes, FITC-conjugated goat polyclonal anti-rabbit immunoglobulin G-H\&L secondary antibody [1:100; Abcam (Hong Kong) Ltd.; cat. no. ab97050] was added to the slides and incubated for $30 \mathrm{~min}$. The slides were rinsed three times, and were incubated with 4',6-diamidino-2-phenylindole for $5 \mathrm{~min}$. Following a further three washes with PBS, the specimens were sealed with glycerin and observed under an inverted fluorescence microscope (ECLIPSE TE2000-S).

Cell Counting kit (CCK)-8 cell proliferation assay. In order to determine the influence of DMOG on BM-MSC proliferation, NBM-MSCs and DBM-MSCs were trypsinized, neutralized, and centrifuged at $100 \mathrm{x}$ g for $6 \mathrm{~min}$. Resuspended in fresh complete medium, a $200 \mu \mathrm{l}$ cell suspension was added to 96 -well plates at a density of $2 \times 10^{3}$ cells/well. After $24 \mathrm{~h}$, $20 \mu$ l CCK-8 reagent (CCK-8; Dojindo Laboratories, Inc., Kumamoto, Japan) was added to the five experimental wells for 2 h. Subsequently, a microplate reader (SpectraMax Plus384; Molecular Devices, LLC, Sunnyvale, CA, USA) was used to detect optical density (OD) values at $450 \mathrm{~nm}$ wavelength. In addition, a blank control well was set containing only $200 \mu \mathrm{l}$ culture medium. OD values were determined for 7 consecutive days at the same time-point.

Cell migration assay. In order to determine the effects of DMOG on BM-MSCs migration capacity, a Transwell assay was conducted. NBM-MSCs and DBM-MSCs were resuspended in DMEM containing 2\% FBS. Briefly, $600 \mu \mathrm{l}$ culture medium containing $150 \mathrm{ng} / \mathrm{ml}$ stromal cell-derived factor- $1 \alpha$ (SDF-1 $\alpha$; PeproTech) was placed into the lower chamber of the 24 -well plate, and $150 \mu \mathrm{l}$ cell suspension $\left(1 \times 10^{4}\right.$ cells $\left./ \mathrm{ml}\right)$ was plated into the upper chamber of the 24-well plate that contained Transwell inserts (diameter, $6.5 \mathrm{~mm}$; pore size, $8 \mu \mathrm{m}$; Corning, Inc., Corning, NY, USA) . Following a $15 \mathrm{~h}$ incubation, the inside compartments were removed and a cotton swab was used to remove the cells. Following fixation with $4 \%$ paraformaldehyde, crystal violet staining and flushing with double distilled water, the cells that had migrated to the lower chamber were observed under a microscope. The migrated cells were counted in five random microscope fields (ECLIPSE TE2000-S).

Enzyme-linked immunosorbent assay (ELISA). An ELISA was performed to analyze the paracrine capacity of the cells. NBM-MSCs and DBM-MSCs were plated in 12-well plates at a density of $5 \times 10^{4}$ cells/well. The medium was discarded after $24 \mathrm{~h}$ and the cells were washed twice with PBS. Subsequently, 
A

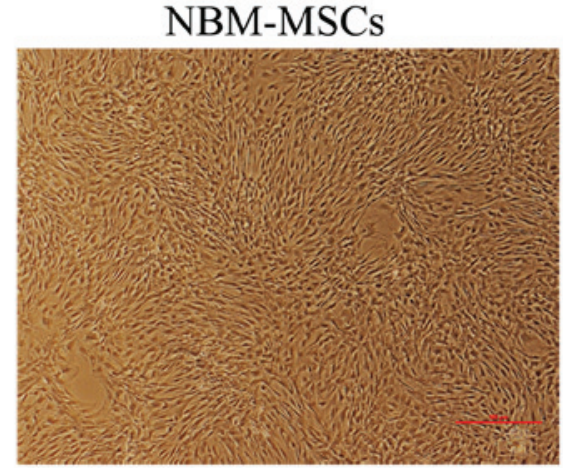

DBM-MSCs

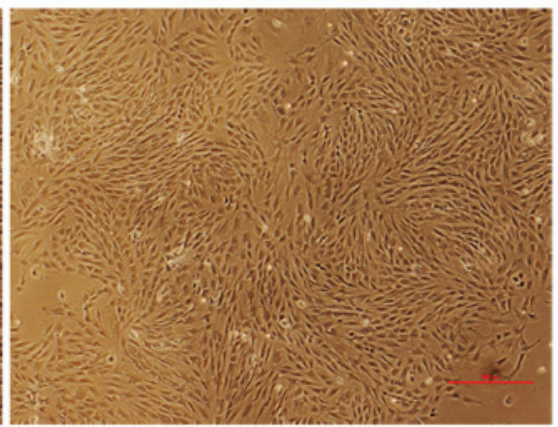

B
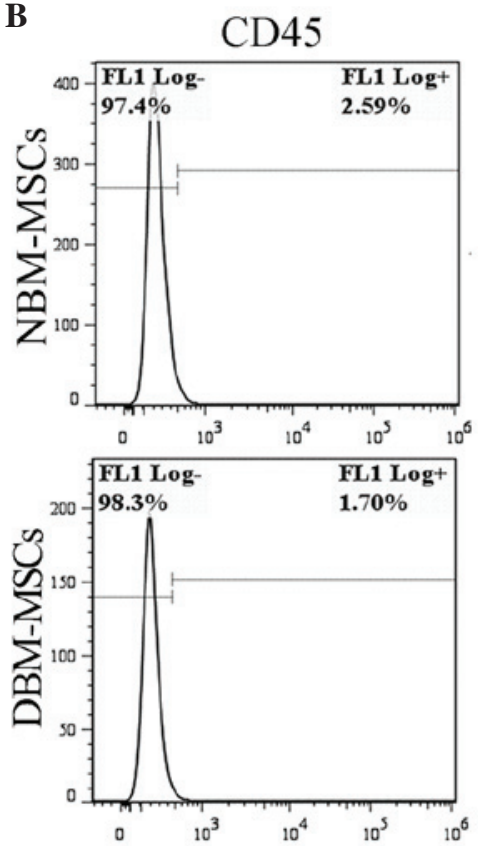

CD44


CD90
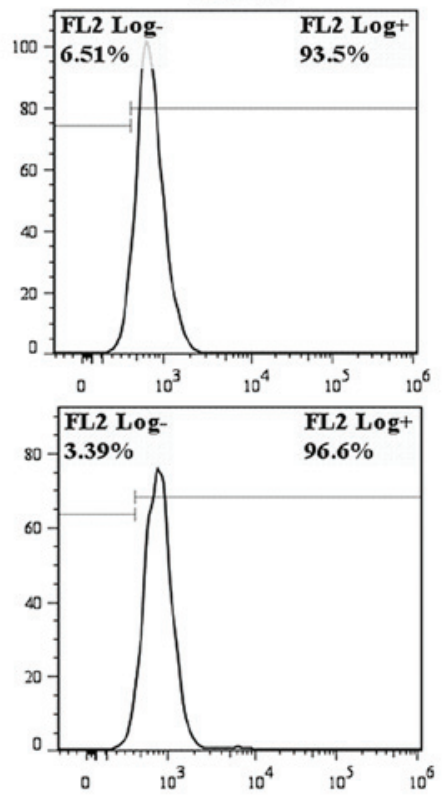

Figure 1. Morphology and immune phenotype of NBM-MSCs and DBM-MSCs. (A) NBM-MSCs and DBM-MSCs from passage 3 were observed under an inverted fluorescence microscope. Scale bar, $500 \mu \mathrm{m}$. (B) Surface antigen markers on passage 3 NBM-MSCs and DBM-MSCs were detected by flow cytometry. NBM-MSCs and DBM-MSCs expressed CD44 (93.8 and 97.9\%, respectively) and CD90 (93.5 and $96.6 \%$, respectively), but not CD45 (2.59 and $1.70 \%$, respectively). Experiments were performed in triplicate, and there was no significant difference between them (P>0.05). NBM-MSCs, bone marrow-derived mesenchymal stem cells from normal saline-treated mice; DBM-MSCs, bone marrow-derived mesenchymal stem cells from dimethyloxallyl glycine-preconditioned mice; CD, cluster of differentiation.

$500 \mu 1$ serum-free DMEM-LG was added and the supernatants were collected after $48 \mathrm{~h}$. TGF and platelet-derived growth factor (PDGF) concentration was detected using corresponding ELISA kits. Mouse TGF ( $\beta$ IG-H3) and PDGF ELISA kits (Wuhan Boster Biological Co., Ltd., Wuhan, China) were used, according to the manufacturer's protocol.

Statistical analysis. All statistical analyses were performed using SPSS 19.0 statistical software (SPSS IBM, Armonk, NY, USA). Experiments were repeated at least three times and data are presented as the mean \pm standard deviation. Student's two-tailed t-test was used to compare the two independent experimental groups. $\mathrm{P}<0.05$ was considered to indicate a statistically significant difference.

\section{Results}

Cell morphology, immune phenotype and multilineage differentiation ability is similar in DBM-MSCs and NBM-MSCs.
To detect the immune phenotype and differentiation potential of the cells, NBM-MSCs and DBM-MSCs were cultured in parallel. The homogeneous layer of fibroblast-like BM-MSCs obtained from DMOG-preconditioned mice was similar to that obtained from NS-treated mice (Fig. 1A).

The cell surface antigen expression of NBM-MSCs and DBM-MSCs was analyzed by flow cytometry for three samples. The two types of cells were positive for CD44 (homing-associated cell adhesion molecule; 93.8 and $97.9 \%$, in NBM-MSCs and DBM-MSCs, respectively) and CD90 (Thy-1; 93.5 and 96.6\% in NBM-MSCs and DBM-MSCs, respectively), but were negative for hematopoietic stem cell marker CD45 (leukocyte common antigen; 2.59 and 1.70\% in NBM-MSCs and DBM-MSCs, respectively; Fig. 1B). These results indicate that DBM-MSCs possess a similar immune phenotype to NBM-MSCs. In addition, no significant differences were detected between them $(\mathrm{P}>0.05)$.

NBM-MSCs and DBM-MSCs were separately cultured in adipogenic, osteogenic or chondrogenic induction medium 
A



$\mathbf{x 1 0 0}$

B
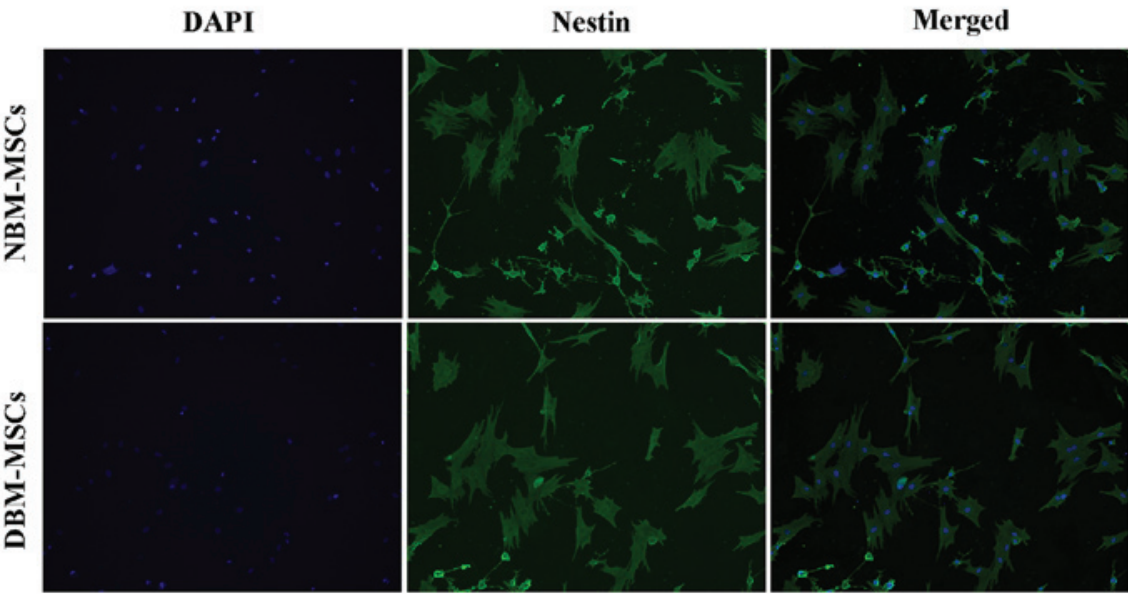

$\mathbf{x 2 0 0}$

Figure 2. Multilineage differentiation capacity of NBM-MSCs and DBM-MSCs. (A) NBM-MSCs and DBM-MSCs were cultured in Ad., Os., or Ch. differentiation medium. Adipogenic differentiation was stained with Oil Red O. Osteogenic differentiation was stained with Von Kossa. Chondrogenic differentiation was stained with Toluidine Blue. (Magnification, x100). (B) NBM-MSCs and DBM-MSCs were induced into neuron-like cells. Immunofluorescence staining: Nuclei were dyed blue with DAPI and Nestin expression was dyed green. (Magnification, x200). NBM-MSCs, bone marrow-derived mesenchymal stem cells from normal saline-treated mice; DBM-MSCs, bone marrow-derived mesenchymal stem cells from dimethyloxallyl glycine-preconditioned mice; Ad., adipogenic; Os., osteogenic; Ch., chondrogenic; DAPI, 4',6-diamidino-2-phenylindole.

for 14-21 days. Subsequently, the presence of cytoplasmic olesomes, the formation of calcium precipitation, or the secretion of acid glycosaminoglycan was examined by specific staining. NBM-MSCs and DBM-MSCs were able to be differentiated into adipocytes that contained secreting orange-red lipid droplets, osteoblasts that are surrounded by black calcium precipitation and chondrocytes that have cell matrix that dyes purple and nuclei that stain blue (Fig. 2A). In addition, DBM-MSCs induced by $\beta$-mercaptoethanol for $5 \mathrm{~h}$ were immunocytochemically shown to display typical neuron-like characteristics, and express nerve cell-specific marker Nestin, but not astrocyte-specific marker GFAP (Fig. 2B; data not shown). These results suggest that the multilineage differentiation capacity of DBM-MSCs was similar to that of NBM-MSCs.

Cell proliferation is slightly reduced in DBM-MSCs compared with NBM-MSCs. To determine the influence of DMOG on MSCs proliferation, the number of MSCs derived from the two

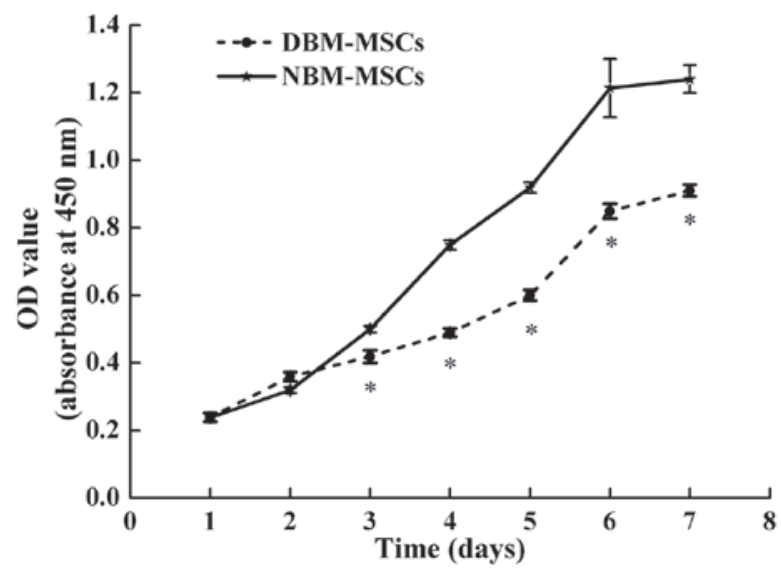

Figure 3. Cell proliferation was detected using the CCK- 8 assay. There were significant differences from day 3 to day 7 between the NBM-MSCs and DBM-MSCs. Data are presented as the mean \pm standard deviation. ${ }^{*} \mathrm{P}<0.05$. CCK-8, Cell Counting kit-8; BM-MSCs, bone marrow-derived mesenchymal stem cells from normal saline-treated mice; DBM-MSCs, bone marrow-derived mesenchymal stem cells from dimethyloxallyl glycine-preconditioned mice; OD, optical density. 
A

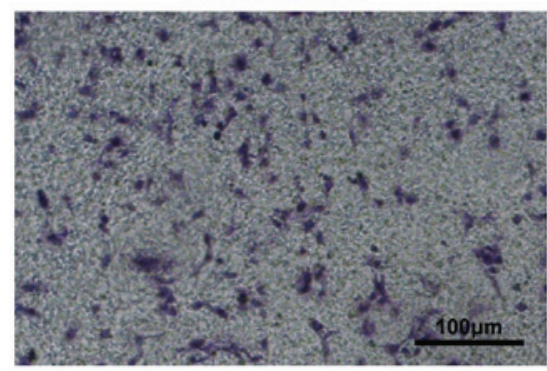

DBM-MSCs

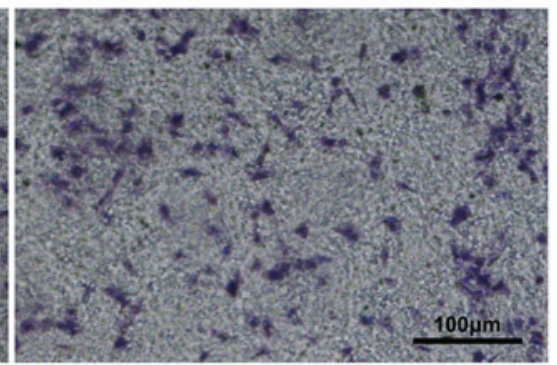

B

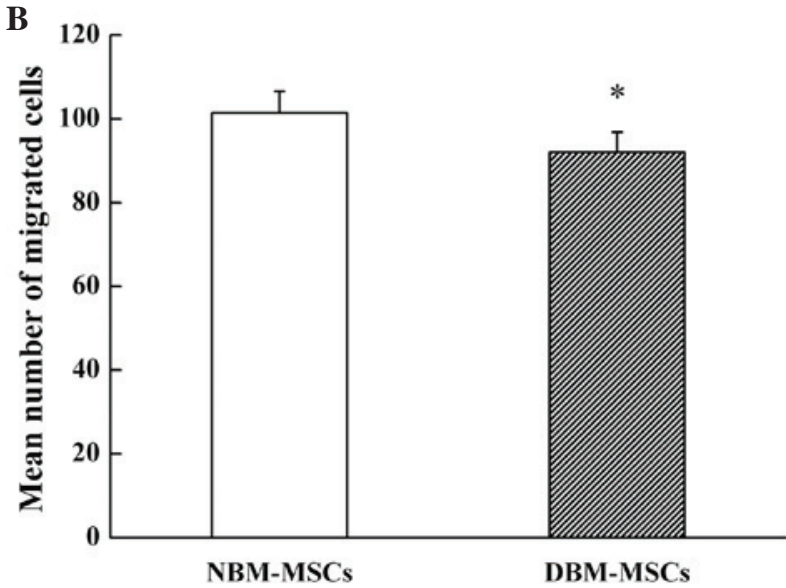

Figure 4. Migratory ability of NBM-MSCs and DBM-MSCs to chemokine SDF-1 $\alpha$. (A) Migration of NBM-MSCs and DBM-MSCs induced by SDF-1 $\alpha$ was detected using a Transwell assay. Migrated cells were stained with crystal violet. Scale bar, $100 \mu \mathrm{m}$. (B) Quantification of migration results. Data are presented as the mean \pm standard deviation. ${ }^{*} \mathrm{P}<0.05$. BM-MSCs, bone marrow-derived mesenchymal stem cells from normal saline-treated mice; DBM-MSCs, bone marrow-derived mesenchymal stem cells from dimethyloxallyl glycine-preconditioned mice; SDF-1 $\alpha$, stromal cell-derived factor-1 $\alpha$.

groups was measured using the CCK-8 test. DBM-MSCs were in a latent period of growth between days 1 and 3, exhibited logarithmic growth between days 4 and 6 , and reached a plateau after day 6. Compared with NBM-MSCs, the proliferation rate of DBM-MSCs was reduced, and starting from day 3 the total number of cells was significantly decreased compared with the number of NBM-MSCs at the same time $(\mathrm{P}=0.003$, $\mathrm{P}=0.001, \mathrm{P}=0.004, \mathrm{P}=0.007$ and $\mathrm{P}=0.003$ for day 3 to day 7 , respectively). The results also indicated that DBM-MSCs had a similar latent period, logarithmic phase and plateau to the NBM-MSCs (Fig. 3). Both cell growth curves were 'S'-shaped; however, the proliferative ability of DBM-MSCs was slightly reduced compared with NBM-MSCs.

Migratory ability is slightly reduced in DBM-MSCs compared with NBM-MSCs. The Transwell assay was used to compare differences in migratory ability between DBM-MSCs and NBM-MSCs. As shown in Fig. 4A, the migrated cells from the two groups were detected (Fig. 4A). Results indicated that following $15 \mathrm{~h}$ of SDF- $1 \alpha$ as a chemoattractant, the number of DBM-MSCs that had migrated to the lower compartment was reduced compared with the number of NBM-MSCs

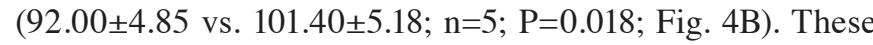
results suggest that the migratory ability of DBM-MSCs was inferior to that of NBM-MSCs.

DBM-MSCs exhibit a similar TGF and PDGF secretory capacity compared with NBM-MSCs. In order to determine whether cytokine production was affected by DMOG, the
TGF and PDGF cytokine concentrations were detected in DBM-MSCs and NBM-MSCs by ELISA. Compared with NBM-MSCs, TGF secretion of DBM-MSCs was decreased; however, there was no significant difference between the groups $(689.9 \pm 40.2$ vs. $746.4 \pm 43.8 \mathrm{ng} / \mathrm{ml} ; \mathrm{P}=0.066$; Fig. 5A). Conversely, PDGF secretion of DBM-MSCs was increased; however, this difference was also not significant $(1323.5 \pm 110.3$ vs. $1207.9 \pm 98.7 \mathrm{ng} / \mathrm{ml} ; \mathrm{P}=0.119$; Fig. $5 \mathrm{~B})$. These results indicate that DBM-MSCs have a similar secretory capacity with regards to TGF and PDGF, as compared with NBM-MSCs.

\section{Discussion}

Our previous study revealed that MSCs could be mobilized into peripheral blood circulation by hypoxia induction, and the transcription factor HIF-1 $\alpha$ had a pivotal role in hypoxia-induced MSCs mobilization (31). Considering clinical and ethical safety, hypoxia mobilization is not feasible in clinical therapy. However, it has been reported that similar effects may be obtained using the prolyl hydroxylase inhibitor DMOG (32), which is a type of hypoxia-mimetic agent that stabilizes and upregulates HIF-1 signaling under normoxic conditions.

The present study investigated the biological properties of BM-MSCs obtained from ICR mice preconditioned with DMOG. Previous studies have mainly investigated the effects of DMOG by directly preconditioning stem cells in vitro, in order to assess the benefits $(3,8)$. However, in the present study, 

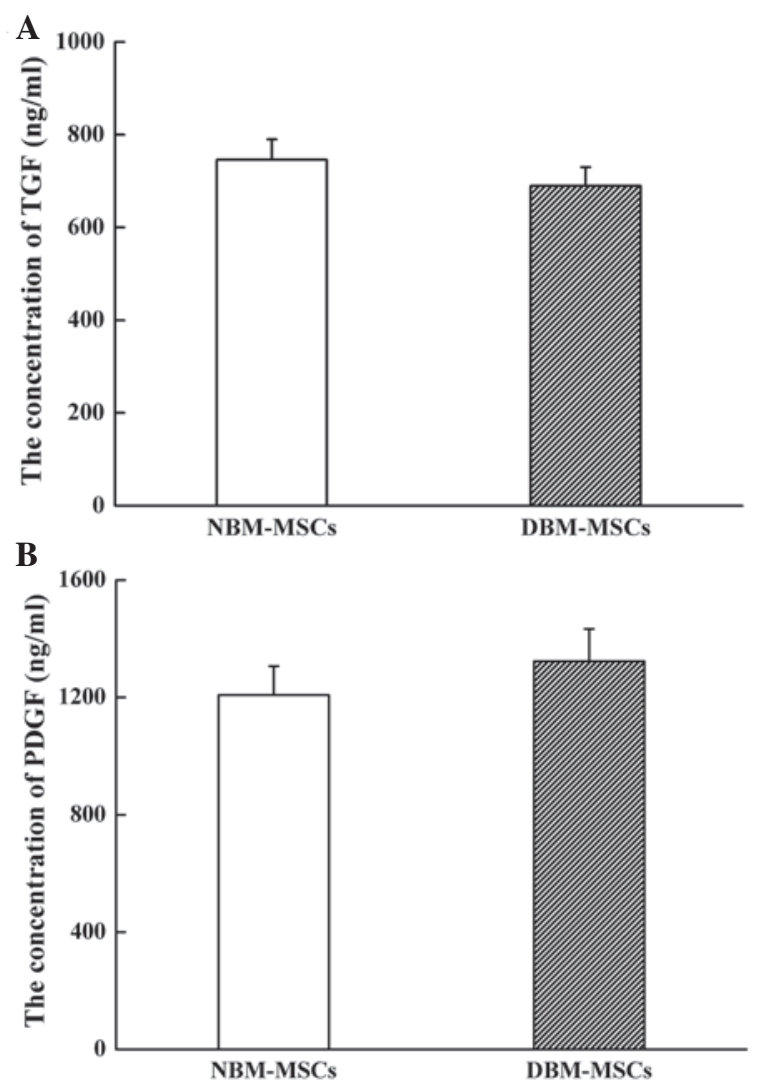

Figure 5. Paracrine capacity of NBM-MSCs and DBM-MSCs. (A) Quantification of TGF concentration in the culture supernatant of NBM-MSCs and DBM-MSCs. There was no significant difference between the expression levels $(\mathrm{P}>0.05)$. (B) Quantification of PDGF concentration in the culture supernatant of NBM-MSCs and DBM-MSCs. No significant difference was identified between them $(\mathrm{P}>0.05)$. TGF and PDGF concentrations were detected using enzyme-linked immunosorbent assays. Data are presented as the mean \pm standard deviation. BM-MSCs, bone marrow-derived mesenchymal stem cells from normal saline-treated mice; DBM-MSCs, bone marrow-derived mesenchymal stem cells from dimethyloxallyl glycine-preconditioned mice; TGF, transforming growth factor; PDGF, platelet-derived growth factor.

a DMOG preconditioning strategy was used, which differs from the methods used in previous studies. The present study detected the effects of DMOG on BM-MSCs by collecting cells from mice that were intraperitoneally injected with DMOG.

At present, no specific surface markers of MSCs have been identified. Previous studies have indicated that the surface antigen phenotype of MSCs is not singular, but possesses the characteristics of mesenchymal cells, epithelial cells and muscle cells at the same time $(33,34)$. MSCs are negative for hematopoietic cell surface antigens, including CD34, CD45, CD11, CD14 and CD235a, and adhesion molecules, such as CD31, CD18 and CD56. Conversely, MSCs are positive for CD105, CD73, CD90, CD71, CD29, CD44, CD106, CD166, etc. (35). The results of the present study revealed that DBM-MSCs and NBM-MSCs were negative for CD45, and positive for CD44 and CD90, thus suggesting that DBM-MSCs exhibit a similar immune phenotype to NBM-MSCs, as expected.

MSCs can be expanded in vitro and maintain multilineage differentiation potential (36). Detection of adipogenic, osteogenic, chondrogenic and neuronal differentiation potential is the most common method used to identify whether analyzed cell populations are capable of multilineage differentiation. Wnt and Rho are the main signaling pathways associated with regulation of adipogenic differentiation of MSCs (37). Adipogenic stimuli induce terminal differentiation of committed preadipocytes via the epigenomic activation of peroxisome proliferator-activated receptor- $\gamma$ (PPAR $\gamma)$. The coordination of PPAR $\gamma$ with CCAAT/enhancer-binding protein transcription factors is able to maintain adipocyte gene expression (38). In addition, Wnt, Notch and bone morphogenetic protein signaling has an important role in the regulation of MSCs osteogenic differentiation (39). DMOG has been reported to increase the bone healing capacity of adipose-derived MSCs by promoting osteogenic differentiation and angiogenic potential in rat critical-sized calvarial defects (3). In addition, a previous study suggested that TGF- $\beta$ signals have a pivotal role in chondrogenic differentiation (40). Hypoxia-enhanced chondrogenesis of BM-MSCs has also been reported to occur via activation of the mitogen-activated protein kinase P38 pathway (41). Furthermore, sirtuin 1 activation may be essential for the induction of neuronal differentiation, due to its effects on mammalian target of rapamycin downregulation and neurite outgrowth stimulation (42). In the present study, compared with NBM-MSCs, DBM-MSCs exhibited similar adipogenic, osteogenic, chondrogenic and neuronal differentiation abilities, thus suggesting that DMOG had no obvious stimulatory or inhibitory effects on BM-MSCs multilineage differentiation. While previous studies have predominantly investigated the effects of DMOG by directly preconditioning stem cells in vitro $(3,8)$, the present study investigated the biological properties of BM-MSCs obtained from ICR mice preconditioned with DMOG. By contrast to in vitro treatment, the current study hypothesizes the in vivo treatment would be influenced by complex metabolic reactions in the animal body. No notable change in the differentiation ability of BM-MSCs was observed in the present in vitro culture and our previous study also demonstrated DMOG could mobilize MSCs to the peripheral blood with no effect on differentiation in pretreated mice (28). Thus, DMOG appears to be feasible as a stem cell mobilization agent.

A cell growth curve was generated using the CCK-8 assay, and the proliferative ability of DBM-MSCs was slightly reduced, compared with that of NBM-MSCs. These results suggested that DMOG slightly inhibited BM-MSCs proliferation. However, DMOG treatment maintained a normal growth curve, thus suggesting that DMOG had no obvious cytotoxic effects on BM-MSCs as only a slightly reduced proliferation was observed. This result is similar to the findings of a previous study on the effects of DMOG on adipose-derived MSCs (3). In the in vivo microenvironment, MSCs constantly update themselves, with the majority of cells maintained in the latent period of growth. There are few studies that have reported the effects of DMOG on MSCs proliferation. A previous study demonstrated that DMOG was able to inhibit the proliferation of vascular smooth muscle cells in vitro (43). Furthermore, it has been reported that DMOG may significantly reduce the apoptosis of MSCs, stabilize the expression of HIF-1 $\alpha$ to induce glucose transport protein synthesis, and reduce the release of mitochondrial cytochrome $c$, thus promoting protein kinase phosphorylation (44). Therefore, the reduction in the proliferative ability of DBM-MSCs may be associated with the varying expression levels of proteins involved in cell cycle regulation. 
The present study detected the migratory capacity of DBM-MSCs, and SDF-1 $\alpha$ was used as a chemotaxin. The results demonstrated that DBM-MSCs possessed weaker migratory ability compared with NBM-MSCs. Our research group and others have revealed that the SDF-1 $\alpha / \mathrm{CXC}$ chemokine receptor (CXCR) axis has an important role in mediating MSCs migration $(45,46)$. In addition, Wang et al $(47)$ indicated that CXCR-4 and CXCR-7 receptors were co-expressed in BM-MSCs and synergistically promoted BM-MSC migration. However, the in vitro migration assay employed in the present study may not directly mimic the in vivo conditions necessary for BM-MSCs migration. Hu et al (48) demonstrated that pretreatment of the BM-MSCs with the CXCR4 antagonist AMD3100 significantly inhibited the mobilization of BM-MSCs in vitro and in vivo. Therefore, the decreased migratory capacity of DBM-MSCs may be associated with reduced CXCR expression.

Paracrine capacity is one of the main mechanisms by which MSCs exert their functions on damage repair, blood vessel formation and blood supply (49). In the present study, TGF and PDGF concentrations were detected in cell culture supernatants. DBM-MSCs exhibited reduced TGF secretion and increased PDGF secretion compared with NBM-MSCs. $\mathrm{Ng}$ et al (50) reported that paracrine TGF- $\beta$ and PDGF signaling was essential for MSCs differentiation and proliferation. TGF- $\beta$ is significantly associated with chondrogenesis, whereas PDGF is significantly associated with adipogenesis and chondrogenesis. Although there were differences in cytokine secretion between DBM-MSCs and NBM-MSCs, no statistical significance was detected.

In conclusion, the results of the present study provide a novel insight into the biological changes of BM-MSCs obtained from mice preconditioned with DMOG. DBM-MSCs were similar in aspects of cell morphology, immune phenotype, multilineage differentiation, and TGF and PDGF secretion, but were slightly distinct with regards to proliferative and migratory capacity compared with NBM-MSCs; however, they may have therapeutic potential for future stem cell therapy. In addition, the present study suggested that DMOG may be used as a novel mobilization agent in future clinical trials as no adverse effects were observed with the mobilization of MSCs to the peripheral blood.

\section{Acknowledgements}

The present study was financially supported by grants from the National Natural Science Foundation of China (grant no. 81270566) and the Medicine and Technology Program of Zhejiang Province (grant no. 2014KYA069).

\section{References}

1. Jaakkola P, Mole DR, Tian YM, Wilson MI, Gielbert J, Gaskell SJ, von Kriegsheim A, Hebestreit HF, Mukherji M, Schofield CJ, et al: Targeting of HIF-alpha to the von Hippel-Lindau ubiquitylation complex by O2-regulated prolyl hydroxylation. Science 292: 468-472, 2001.

2. Ockaili R, Salloum F, Natarajan R, Jones DG, Fisher BJ, Ghosh S, Fowler AA and Kukreja RC: Dimethyloxallyl glycine-A competitive inhibitor of prolyl hydroxylases induces cardioprotective effect via hypoxia inducible factor-1 alpha stabilization in rabbits. Circulation 108: 219-219, 2003.
3. Ding $\mathrm{H}$, Gao YS, Wang $\mathrm{Y}, \mathrm{Hu} \mathrm{C}$, Sun $\mathrm{Y}$ and Zhang CQ: Dimethyloxaloylglycine increases the bone healing capacity of adipose-derived stem cells by promoting osteogenic differentiation and angiogenic potential. Stem Cells Dev 23: 990-1000, 2014.

4. Song YR, You SJ, Lee YM, Chin HJ, Chae DW, Oh YK, Joo KW, Han JS and Na KY: Activation of hypoxia-inducible factor attenuates renal injury in rat remnant kidney. Nephrol Dial Transplant 25: 77-85, 2010.

5. Milkiewicz M, Pugh CW and Egginton S: Inhibition of endogenous HIF inactivation induces angiogenesis in ischaemic skeletal muscles of mice. J Physiol 560: 21-26, 2004.

6. Yuan Q, Bleiziffer O, Boos AM, Sun J, Brandl A, Beier JP, Arkudas A, Schmitz M, Kneser U and Horch RE: PHDs inhibitor DMOG promotes the vascularization process in the AV loop by HIF-1a up-regulation and the preliminary discussion on its kinetics in rat. BMC Biotechnol 14: 112, 2014.

7. Nagel S,Papadakis M,Chen R,Hoyte LC,Brooks KJ, Gallichan D, Sibson NR, Pugh C and Buchan AM: Neuroprotection by dimethyloxalylglycine following permanent and transient focal cerebral ischemia in rats. J Cereb Blood Flow Metab 31: 132-143, 2011.

8. Liu XB, Wang JA, Ji XY, Yu SP and Wei L: Preconditioning of bone marrow mesenchymal stem cells by prolyl hydroxylase inhibition enhances cell survival and angiogenesis in vitro and after transplantation into the ischemic heart of rats. Stem Cell Res Ther 5: 111, 2014.

9. Beyer Nardi N and da Silva Meirelles L: Mesenchymal stem cells: Isolation, in vitro expansion and characterization. Handb Exp Pharmacol 174: 249-282, 2006.

10. Deans RJ and Moseley AB: Mesenchymal stem cells: Biology and potential clinical uses. Exp Hematol 28: 875-884, 2000.

11. Sanchez-Ramos J, Song S, Cardozo-Pelaez F, Hazzi C, Stedeford T, Willing A, Freeman TB, Saporta S, Janssen W, Patel N, et al: Adult bone marrow stromal cells differentiate into neural cells in vitro. Exp Neurol 164: 247-256, 2000.

12. Bianco P, Riminucci M, Gronthos S and Robey PG: Bone marrow stromal stem cells: Nature, biology, and potential applications. Stem Cells 19: 180-192, 2001.

13. Nakagami H, Morishita R, Maeda K, Kikuchi Y, Ogihara T and Kaneda Y: Adipose tissue-derived stromal cells as a novel option for regenerative cell therapy. J Atheroscler Thromb 13: 77-81, 2006.

14. Chunmeng S and Tianmin C: Skin: A promising reservoir for adult stem cell populations. Med Hypotheses 62: 683-688, 2004.

15. De Bari C, Dell'Accio F, Vandenabeele F, Vermeesch JR, Raymackers JM and Luyten FP: Skeletal muscle repair by adult human mesenchymal stem cells from synovial membrane. J Cell Biol 160: 909-918, 2003.

16. Pierdomenico L, Bonsi L, Calvitti M, Rondelli D, Arpinati M, Chirumbolo G, Becchetti E, Marchionni C, Alviano F, Fossati V, et al: Multipotent mesenchymal stem cells with immunosuppressive activity can be easily isolated from dental pulp. Transplantation 80: 836-842, 2005.

17. Yamada Y, Fujimoto A, Ito A, Yoshimi R and Ueda M: Cluster analysis and gene expression profiles: A cDNA microarray system-based comparison between human dental pulp stem cells (hDPSCs) and human mesenchymal stem cells (hMSCs) for tissue engineering cell therapy. Biomaterials 27: 3766-3781, 2006.

18. Lee OK, Kuo TK, Chen WM, Lee KD, Hsieh SL and Chen TH: Isolation of multipotent mesenchymal stem cells from umbilical cord blood. Blood 103: 1669-1675, 2004.

19. Pitchford SC, Hahnel MJ, Jones CP and Rankin SM: Troubleshooting: Quantification of mobilization of progenitor cell subsets from bone marrow in vivo. J Pharmacol Toxicol Methods 61: 113-121, 2010.

20. Alhadlaq A and Mao JJ: Mesenchymal stem cells: Isolation and therapeutics. Stem Cells Dev 13: 436-448, 2004.

21. Lazarus HM, Haynesworth SE, Gerson SL and Caplan AI: Human bone marrow-derived mesenchymal (stromal) progenitor cells (MPCs) cannot be recovered from peripheral blood progenitor cell collections. J Hematother 6: 447-455, 1997.

22. Wexler SA, Donaldson C, Denning-Kendall P, Rice C, Bradley B and Hows JM: Adult bone marrow is a rich source of human mesenchymal 'stem' cells but umbilical cord and mobilized adult blood are not. Br J Haematol 121: 368-374, 2003.

23. Roufosse CA, Direkze NC, Otto WR and Wright NA: Circulating mesenchymal stem cells. Int J Biochem Cell Biol 36: 585-597, 2004. 
24. Neth P, Ciccarella M, Egea V, Hoelters J, Jochum M and Ries C: Wnt signaling regulates the invasion capacity of human mesenchymal stem cells. Stem Cells 24: 1892-1903, 2006.

25. Kumar S and Ponnazhagan S: Mobilization of bone marrow mesenchymal stem cells in vivo augments bone healing in a mouse model of segmental bone defect. Bone 50: 1012-1018, 2012.

26. Deng J, Zou Z, Zhou T, Ai G, Wang J, Dong S and Su S: The mobilization of rat mesenchymal stem cells into peripheral blood by LiCL and its potency differentiation. Chinese Science Bulletin 53: 2632-2638, 2008.

27. Liu W, Yu Q, Liu L, Zhou L and Hu S: Effect of prolylhydroxylase inhibitor on mobilization of mesenchymal stem cells in mice. Zhejiang Zhongyiyaodaxue Xuebao 37: 1371-1376, 2013 (In Chinese)

28. Hu S, Yu Q, Liu L and Ge T: Mechanism of HIF-1 signaling pathway in mediating MSCs mobilization with DMOG. Zhongguo Bijiaoyixue Zazhi 25: 9-14, 2014 (In Chinese).

29. National Research Council: Guide for the care and use of laboratory animals. 7th edition. National Academies Press, Washington, DC, 1996.

30. Campagnoli C, Roberts IA, Kumar S, Bennett PR, Bellantuono I and Fisk NM: Identification of mesenchymal stem/progenitor cells in human first-trimester fetal blood, liver, and bone marrow. Blood 98: 2396-2402, 2001.

31. Liu L, Yu Q, Lin J, Lai X, Cao W, Du K, Wang Y, Wu K, Hu Y, Zhang L, et al: Hypoxia-inducible factor- $1 \alpha$ is essential for hypoxia-induced mesenchymal stem cell mobilization into the peripheral blood. Stem Cells Dev 20: 1961-1971, 2011.

32. Ivan M, Kondo K, Yang H, Kim W, Valiando J, Ohh M, Salic A Asara JM, Lane WS and Kaelin WG Jr: HIFalpha targeted for VHL-mediated destruction by proline hydroxylation: Implications for $\mathrm{O}_{2}$ sensing. Science 292: 464-468, 2001

33. Noort WA, Oerlemans MIFJ, Rozemuller H, Feyen D, Jaksani S, Stecher D, Naaijikens B, Martens AC, Bühring HJ, Doevendans PA and Sluijter JPG: Human versus porcine mesenchymal stromal cells: Phenotype, differentiation potential, immunomodulation and cardiac improvement after transplantation. J Cell Mol Med 16: 1827-1839, 2011

34. Lv FJ, Tuan RS, Cheung KM and Leung VY: Concise review: The surface markers and identity of human mesenchymal stem cells. Stem Cells 32: 1408-1419, 2014

35. Sordi V, Malosio ML, Marchesi F, Mercalli A, Melzi R, Giordano T, Belmonte N, Ferrari G, Leone BE, Bertuzzi F, et al: Bone marrow mesenchymal stem cells express a restricted set of functionally active chemokine receptors capable of promoting migration to pancreatic islets. Blood 106: 419-427, 2005.

36. Pittenger MF, Mackay AM, Beck SC, Jaiswal RK, Douglas R, Mosca JD, Moorman MA, Simonetti DW, Craig S and Marshak DR: Multilineage potential of adult human mesenchymal stem cells. Science 284: 143-147, 1999.

37. Laudes M: Role of WNT signalling in the determination of human mesenchymal stem cells into preadipocytes. J Mol Endocrinol 46: R65-R72, 2011.
38. Cristancho AG and Lazar MA: Forming functional fat: A growing understanding of adipocyte differentiation. Nat Rev Mol Cell Biol 12: 722-734, 2011.

39. Lin GL and Hankenson KD: Integration of BMP, Wnt, and notch signaling pathways in osteoblast differentiation. J Cell Biochem 112: 3491-3501, 2011.

40. Wang W, Li B, Yang J, Xin L, Li Y, Yin H, Qi Y, Jiang Y, Ouyang $\mathrm{H}$ and Gao $\mathrm{C}$ : The restoration of full-thickness cartilage defects with BMSCs and TGF-beta 1 loaded PLGA/fibrin gel constructs. Biomaterials 31: 8964-8973, 2010.

41. Hirao M, Tamai N, Tsumaki N, Yoshikawa H and Myoui A: Oxygen tension regulates chondrocyte differentiation and function during endochondral ossification. J Biol Chem 281: 31079-31092, 2006.

42. Guo W, Qian L, Zhang J, Zhang W, Morrison A, Hayes P, Wilson S, Chen T and Zhao J: Sirtl overexpression in neurons promotes neurite outgrowth and cell survival through inhibition of the mTOR signaling. J Neurosci Res 89: 1723-1736, 2011.

43. Schultz K, Murthy V, Tatro JB and Beasley D: Prolyl hydroxylase 2 deficiency limits proliferation of vascular smooth muscle cells by hypoxia-inducible factor-1\{alpha\}-dependent mechanisms. Am J Physiol Lung Cell Mol Physiol 296: L921-L927, 2009.

44. Liu XB, Wang JA, Ogle ME and Wei L: Prolyl hydroxylase inhibitor dimethyloxalylglycine enhances mesenchymal stem cell survival. J Cell Biochem 106: 903-911, 2009.

45. Yu Q, Liu L, Lin J, Wang Y, Xuan X, Guo Y and Hu S: SDF-1 $\alpha / C X C R 4$ axis mediates the migration of mesenchymal stem cells to the hypoxic-ischemic brain lesion in a rat model. Cell J 16: 440-447, 2015.

46. Peled A, Petit I, Kollet O, Magid M, Ponomaryov T, Byk T, Nagler A, Ben-Hur H, Many A, Shultz L, et al: Dependence of human stem cell engraftment and repopulation of NOD/SCID mice on CXCR4. Science 283: 845-848, 1999.

47. Wang Y, Fu W, Zhang S, He X, Liu Z, Gao D and Xu T: CXCR-7 receptor promotes $\mathrm{SDF}-1 \alpha$-induced migration of bone marrow mesenchymal stem cells in the transient cerebral ischemia/reperfusion rat hippocampus. Brain Res 1575: 78-86, 2014.

48. Hu C, Yong X, Li C, Lü M, Liu D, Chen L, Hu L, Teng M, Zhang D, Fan Y and Liang G: CXCL12/CXCR4 axis promotes mesenchymal stem cell mobilization to burn wounds and contributes to wound repair. J Surg Res 183: 427-434, 2013.

49. Doorn J, Moll G, Le Blanc K, van Blitterswijk C and de Boer J: Therapeutic applications of mesenchymal stromal cells: Paracrine effects and potential improvements. Tissue Eng Part B Rev 18: 101-115, 2012

50. Ng F, Boucher S, Koh S, Sastry KS, Chase L, Lakshmipathy U, Choong C, Yang Z, Vemuri MC, Rao MS and Tanavde V: PDGF, TGF-beta, and FGF signaling is important for differentiation and growth of mesenchymal stem cells (MSCs): Transcriptional profiling can identify markers and signaling pathways important in differentiation of MSCs into adipogenic, chondrogenic, and osteogenic lineages. Blood 112: 295-307, 2008. 